\title{
Crecimiento y supervivencia de procedencias de Enterolobium cyclocarpum en la costa de Oaxaca, México
}

\author{
Growth and survival of Enterolobium cyclocarpum \\ provenances on the coast of Oaxaca, Mexico
}

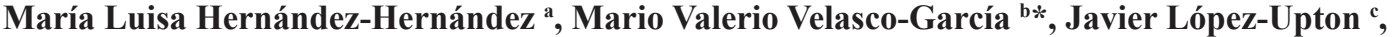 \\ Rolando Galán-Larrea a, Carlos Ramírez-Herrera ${ }^{c}$, Héctor Viveros-Viveros ${ }^{\text {d }}$ \\ ${ }^{a}$ Universidad del Mar, Campus Puerto Escondido, Ciudad Universitaria, Puerto Escondido, Oaxaca, México. \\ *Autor para correspondencia: ${ }^{b}$ Centro Nacional de Investigación Disciplinaria en Conservación y Mejoramiento de Ecosistemas, \\ Instituto Nacional de Investigaciones Forestales, Agrícolas y Pecuarias. Avenida Progreso Número 5, Colonia Barrio Santa Catarina, \\ Delegación Coyoacán, Cuidad de México, tel.: 5538718700, taxodium01@hotmail.com \\ ${ }^{\mathrm{c}}$ Colegio de Postgraduados, Campus Montecillo, Posgrado en Ciencias Forestales, Carretera México-Texcoco km 36.5, Montecillo, \\ Texcoco, Estado de México, México. \\ dUniversidad Veracruzana, Instituto de Investigaciones Forestales. Parque Ecológico "El Haya”, Colonia Benito Juárez, \\ Xalapa, Veracruz, México.
}

\section{SUMMARY}

Enterolobium cyclocarpum is a multiuse-tropical tree whose growth and survival from different origins are not known in specific sites. The objective was to evaluate the differences in initial growth and survival of the provenances between and within the sites and the genotype $\mathrm{x}$ environment interaction in two sites on the coast of Oaxaca, Mexico. The plants were grown in a nursery for six months before planting. Annual periodic increment of basal diameter and height and crown diameter were measured. In addition, the number of bifurcations and survival were determined two years after planting. The differences in precipitation and soil properties (chemical and physical) of the sites caused differences of increment. All the variables had higher values in the site Pinotepa de Don Luis than in Valdeflores, except annual periodic increment of height and survival. In Pinotepa de Don Luis, the trees from local provenance had higher increment of basal diameter, height and crown diameter. In Valdeflores, the closest provenance (Colotepec) showed superior increments in basal diameter and crown diameter. Low values of type B genetic correlation indicated high genotype $\mathrm{x}$ environment interaction. The provenances were more unstable in height growth and number of bifurcations. The genotype $\mathrm{x}$ environment interaction limits the selection of a provenance to establish in both environments; therefore, the provenance to be planted depends on the environment and the objective. The results allow recommending the use of local provenances or those closer to the plantation site.

Key words: annual periodic increment, genotype $\mathrm{x}$ environment interaction, type B genetic correlation, bifurcations, environmental differences.

\section{RESUMEN}

Enterolobium cyclocarpum es un árbol tropical multipropósito, cuyo crecimiento y supervivencia de procedencias distintas en sitios específicos se desconoce. El objetivo fue evaluar las diferencias en crecimiento y supervivencia de 10 procedencias entre y dentro de las localidades y la interacción genotipo x ambiente en dos localidades en la costa de Oaxaca, México. Las plantas se cultivaron en vivero durante seis meses antes de la plantación. Los incrementos periódicos anuales de diámetro basal, altura y diámetro de copa, además del número de bifurcaciones y supervivencia se determinaron dos años después de la plantación. Las diferencias en precipitación y las propiedades del suelo (químicas y físicas) de las localidades ocasionaron diferencias en incremento. Todas las variables tuvieron mayores valores en la localidad Pinotepa de Don Luis que en Valdeflores, excepto el incremento periódico anual de altura y supervivencia. En Pinotepa de Don Luis la procedencia local tuvo mejores incrementos de diámetro basal, altura y diámetro de copa. En Valdeflores, la procedencia la más cercana (Colotepec) presentó mayores incrementos de diámetro basal y diámetro de copa. Los valores bajos de la correlación genética tipo B indicaron interacción genotipo x ambiente alto, y las procedencias fueron más inestables en el crecimiento en altura y número de bifurcaciones. La interacción genotipo x ambiente limita seleccionar una procedencia para establecerse en ambos ambientes; por tanto, la procedencia a plantar dependerá del ambiente y del objetivo. Los resultados permiten recomendar el uso de procedencias locales o más cercanas al sitio de plantación.

Palabras clave: incremento periódico anual, interacción genotipo x ambiente, correlación genética tipo B, bifurcaciones, diferencias ambientales. 


\section{INTRODUCCIÓN}

Enterolobium cyclocarpum (Jacq.) Griseb. es un árbol tropical con distribución natural desde México al Norte de Sudamérica, Jamaica, Cuba, Trinidad y Guyana (Vázquez-Yanes et al. 1999). En México, se distribuye por la vertiente del pacífico, de Sinaloa a Chiapas, y por el del Golfo de México, de Tamaulipas a Campeche (Pennington y Sarukhán 2005). Crece en selvas altas perennifolias, medianas subcaducifolias, medianas subperennifolias y bajas caducifolias (Salas-Morales et al. 2003, Pennington y Sarukhán 2005).

En áreas ganaderas y agrícolas se planta E. cyclocarpum para sombra (Pennington y Sarukhán 2005). La madera de E. cyclocarpum es resistente a la pudrición y al ataque de insectos, por lo que se utiliza para muebles, puertas, cubierta de paredes, canoas, ruedas de carretas, pulpa para papel, aglomerados, instrumentos musicales, utensilios de cocina y artesanías (Pennington y Sarukhán 2005). Las semillas poseen concentraciones altas de minerales, carbohidratos y proteínas, por lo que tienen potencial para uso alimenticio, industrial, medicinal y químico (BarrientosRamírez et al. 2015).

En México, E. cyclocarpum se planta para restaurar sitios degradados, en sistemas agroforestales y con fines comerciales (Vázquez-Yanes et al. 1999, Jiménez-Pascual 2011); sin embargo, la madera que se usa actualmente proviene de relictos de selva, lo que ha provocado poblaciones fragmentadas, con árboles aislados y densidades de 0,1 árboles $\mathrm{ha}^{-1}$, en áreas ganaderas, orillas de caminos y ríos (Salas-Morales et al. 2003, Olivares-Pérez et al. 2011). Debido a esto, E. cyclocarpum puede estar en riesgo, por lo que es necesario establecer plantaciones forestales. Para esto se debe generar conocimiento sobre el crecimiento y la adaptación de diversas fuentes de germoplasma (procedencias) en localidades con ambientes distintos, que permita recomendar el material genético adecuado en cada sitio de plantación para aumentar el éxito de las plantaciones que se realicen. Una forma de obtener este conocimiento es evaluar la adaptación y la productividad de plantas en ensayos de procedencias establecidos en las diferentes zonas de interés (White et al. 2007).

En México se ha estudiado el crecimiento en diámetro y altura, así como la supervivencia en Nayarit, Nuevo León y Colima (Makocki y Valdez-Hernández 2001, Foroughbakhch et al. 2006, Jiménez-Pascual 2011). Sin embargo, se carece de información sobre el crecimiento y adaptación de plantas procedentes de la costa de Oaxaca, México, donde solo existe información sobre tratamientos pre-germinativos y variación física de semillas, variación geográfica de la germinación y crecimiento inicial en vivero (Quino-Pascual 2013, Viveros-Viveros et al. 2015, 2017). Tampoco existen estudios que indiquen el comportamiento de las plantas de diferentes procedencias establecidas en varios sitios de plantación (interacción genotipo $\mathrm{x}$ ambiente). Las semillas de procedencias locales son garantía de adaptación y la mejor opción para establecer plantaciones forestales mientras se desconozca información derivada de estudios de procedencias o progenies (White et al. 2007). Lo anterior es importante cuando las condiciones ambientales de la distribución de las especies y de los sitios para plantar son variables, como se demuestra con algunas de coníferas y latifoliadas (Schenone et al. 2002, Sierra-Lucero et al. 2002, Lozada et al. 2003, López-Upton et al. 2004, Mora-Poblete y Meneses-Rojas 2004, Viveros-Viveros et al. 2005, Valencia-Manzo et al. 2006, Craven et al. 2007). Por tanto, el conocimiento generado en la presente investigación será útil para demostrar que el origen del germoplasma de E. cyclocarpum es importante en la producción de plantas y aumentar el éxito de las plantaciones en los sitios de interés.

Por lo anterior, los objetivos de la presente investigación son determinar la variación del crecimiento y supervivencia en campo de planta procedente de la costa de Oaxaca, México; así como conocer la magnitud y el tipo de interacción genotipo $\mathrm{x}$ ambiente de estas características de árboles de distintas procedencias plantados en dos localidades. La hipótesis de investigación es que el crecimiento y supervivencia de las plantas de distintas procedencias de E. cyclocarpum son diferentes debido a que las condiciones ambientales y de suelos a las que se han adaptado en su sitio de origen son variables, esperando que las plantas de procedencias cercanas a los sitios de plantaciones presentarán mayores tasas de crecimiento y supervivencia. Además, plantas de la misma procedencia tienen crecimiento y supervivencia diferentes en cada localidad de plantación debido a la interacción genotipo por ambiente.

\section{MÉTODOS}

Recolección, beneficio y almacenamiento de semillas. Los frutos se recolectaron de marzo a mayo del 2008 en árboles (de siete a 12 por procedencia) con características deseables, libres de plagas y enfermedades, en 10 procedencias en la región Costa de Oaxaca (figura 1, cuadro 1). Los frutos se transportaron en costales etiquetados con datos de la procedencia y la fecha de recolecta. Para el beneficio de semillas los frutos se secaron al sol, posteriormente, estos se colocaron en costales los cuales se golpearon con una vara para romper los frutos; después, manualmente se separaron las semillas vanas, trozos de material vegetal y piedras. Las semillas limpias se almacenaron durante seis meses a temperatura ambiente en bolsas de plástico obscuro etiquetadas previamente.

Germinación y producción de plantas. Para homogeneizar e incrementar la velocidad de germinación, la testa de las semillas se degradó con una lija (Viveros-Viveros et al. 2015). Después, las semillas de cada procedencia se germinaron en almácigos con sustrato constituido por $35 \%$ de suelo de monte, $35 \%$ de corteza y $30 \%$ de aserrín compostados. 
Las plántulas, después de la caída de los cotiledones, se trasplantaron en bolsas de polietileno de $15 \mathrm{~cm}$ de diámetro por $26 \mathrm{~cm}$ de alto $\left(4.595 \mathrm{~cm}^{3}\right)$, con el mismo tipo de sustrato que se usó para la germinación. El cultivo de plantas se realizó en vivero durante seis meses, con

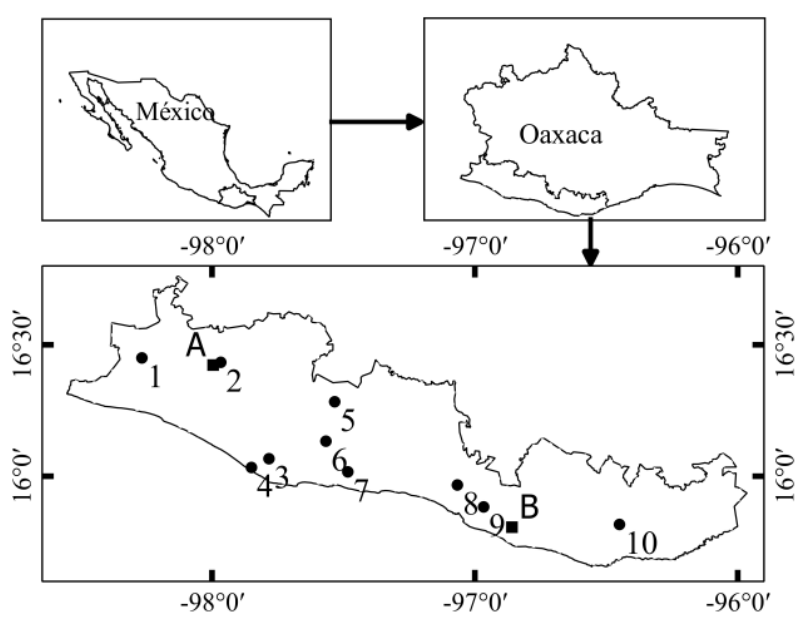

Figura 1. Ubicación de las procedencias (1: Cortijo, 2: Pinotepa de Don Luis, 3: El Zarzal, 4: La Tuza, 5: Tataltepec, 6: San Francisco, 7: Los Limones, 8: San Pedro, 9: Colotepec, 10: Pochutla) de Enterolobium cyclocarpum evaluadas en dos localidades (A: Pinotepa de Don Luis, B: Valdeflores) de la costa de Oaxaca, México.

Geographical location of provenances (1: Cortijo, 2: Pinotepa de Don Luis, 3: El Zarzal, 4: La Tuza, 5: Tataltepec, 6: San Francisco, 7: Los Limones, 8: San Pedro, 9: Colotepec, 10: Pochutla) of Enterolobium cyclocarpum in two sites (A: Pinotepa de Don Luis, B: Valdeflores) of the coast of Oaxaca, Mexico. riegos cada tercer día y la fertilización foliar cada semana; esta última con fórmula y dosis diferentes de acuerdo con la fase de crecimiento (Aldama-Barajas y Aguilera-Rodríguez 2003). Durante las dos últimas semanas del cultivo, para inducir el endurecimiento de sus tallos, las plantas se expusieron al sol y la frecuencia de riego disminuyó al $50 \%$. Al término del cultivo, las plantas con diámetro mínimo de $1 \mathrm{~cm}$, altura mínima de $25 \mathrm{~cm}$, tallo lignificado, sanas y vigorosas se seleccionaron para la plantación.

Establecimiento de los ensayos y diseño experimental. El ensayo de procedencias se estableció en junio y julio de 2009 en Valdeflores y Pinotepa de Don Luis (localidades con condiciones contrastantes), región Costa de Oaxaca, México (figura 1, cuadro 2). En cada localidad se utilizó el diseño experimental de bloques completamente al azar, con 10 tratamientos (procedencias), seis repeticiones conformadas por seis bloques y cuatro plantas por unidad experimental. Para evitar el efecto de borde se plantaron dos líneas de plantas alrededor del experimento. En ambas localidades, antes de la plantación se eliminaron las herbáceas, luego se cavaron cepas comunes (de 40 x 40 x $40 \mathrm{~cm}$ ) con separación de $5 \mathrm{~m}$ entre plantas y líneas de plantación. Durante dos años, el control manual de la vegetación herbácea se realizó cada vez que se requirió. En cada localidad se obtuvieron nueve muestras de suelo en nueve pozos ( 40 x $40 \mathrm{~cm}$ de superficie y $60 \mathrm{~cm}$ de profundidad) distribuidos en forma sistemática. Cada muestra se secó y se cribó en una malla con abertura de $3 \times 3 \mathrm{~mm}$, después se obtuvo una muestra compuesta de cada localidad; el análisis físico y químico (cuadro 2) de estas se realizó en el Laboratorio de Análisis Agrícola de la Costa, Oaxaca conforme a la Norma Oficial Mexicana NOM-021-RECNAT-2010 (SEMARNAT 2010).

Cuadro 1. Ubicación y características de las procedencias de Enterolobium cyclocarpum evaluadas en la costa de Oaxaca, México.

Geographical location and characteristics of the provenances of Enterolobium cyclocarpum evaluated on the coast of Oaxaca, Mexico.

\begin{tabular}{|c|c|c|c|c|c|c|}
\hline Procedencia & $\begin{array}{l}\text { Longitud } \\
(\mathrm{O})\end{array}$ & $\begin{array}{l}\text { Latitud } \\
(\mathrm{N})\end{array}$ & $\begin{array}{l}\text { Altitud } \\
(\mathrm{m})\end{array}$ & $\begin{array}{l}\text { Temperatura media } \\
\text { anual }\left({ }^{\circ} \mathrm{C}\right)^{\dagger}\end{array}$ & $\begin{array}{l}\text { Precipitación media } \\
\text { anual }(\mathrm{mm})^{\dagger}\end{array}$ & Tipo de suelo \\
\hline Cortijo & $98^{\circ} 16^{\prime}$ & $16^{\circ} 27^{\prime}$ & 59 & 28,5 & 1.031 & Cambisol, Fhaozem \\
\hline Pinotepa de Don Luis ${ }^{\dagger \dagger}$ & $97^{\circ} 58^{\prime}$ & $16^{\circ} 26^{\prime}$ & 420 & 27,7 & 1.446 & Regosol \\
\hline El Zarzal & $97^{\circ} 47^{\prime}$ & $16^{\circ} 04^{\prime}$ & 14 & 28,4 & 1.069 & Phaeozem, Regosol \\
\hline La Tuza & $97^{\circ} 51^{\prime}$ & $16^{\circ} 02^{\prime}$ & 15 & 28,4 & 1.064 & Gleysol \\
\hline Tataltepec & $97^{\circ} 32^{\prime}$ & $16^{\circ} 17^{\prime}$ & 370 & 28,1 & 1.140 & Regosol \\
\hline San Francisco & $97^{\circ} 34^{\prime}$ & $16^{\circ} 08^{\prime}$ & 67 & 28,5 & 1.083 & Phaeozem \\
\hline Los Limones & $97^{\circ} 29^{\prime}$ & $16^{\circ} 01^{\prime}$ & 23 & 28,5 & 1.070 & Phaeozem \\
\hline San Pedro & $97^{\circ} 04^{\prime}$ & $15^{\circ} 58^{\prime}$ & 240 & 27,5 & 985 & Phaeozem, Regosol \\
\hline Colotepec & $96^{\circ} 58^{\prime}$ & $15^{\circ} 53^{\prime}$ & 37 & 27,9 & 840 & Phaeozem, Cambisol \\
\hline Pochutla & $96^{\circ} 27^{\prime}$ & $15^{\circ} 49^{\prime}$ & 234 & 27,0 & 1.168 & Cambisol, Regosol \\
\hline
\end{tabular}

† Obtenido de Viveros-Viveros et al. (2017). ${ }^{\dagger}$ Esta procedencia es local para la localidad de ensayo del mismo nombre (cuadro 2). 
Cuadro 2. Ubicación y características de las localidades del ensayo de procedencias de Enterolobium cyclocarpum en la costa de Oaxaca, México.

Geographical location and characteristics of the provenances test sites of the Enterolobium cyclocarpum on the coast of Oaxaca, Mexico.

\begin{tabular}{lcc}
\hline \multicolumn{1}{c}{ Ubicación/Características } & $\begin{array}{c}\text { Pinotepa de } \\
\text { Don Luis }\end{array}$ & Valdeflores \\
\hline Longitud O & $97^{\circ} 59^{\prime} 44,8^{\prime \prime}$ & $96^{\circ} 511^{\prime} 32,3^{\prime}{ }^{\circ}$ \\
Latitud N & $16^{\circ} 25^{\prime} 22,4^{\prime \prime}$ & $15^{\circ} 48^{\prime} 22,5^{\prime}$, \\
Altitud (m) & 455 & 90 \\
Temperatura media anual $\left({ }^{\circ} \mathrm{C}\right)$ & 27,7 & 27,0 \\
Precipitación media anual $(\mathrm{mm})$ & 1.442 & 813 \\
Índice de aridez & 0,06 & 0,11 \\
Tipo de suelo & Regosol & Phaeozem
\end{tabular}

Propiedades físicas de suelo:

\begin{tabular}{|c|c|c|}
\hline Arena (\%) & 71,8 & 77,8 \\
\hline Limo (\%) & 14,0 & 6,2 \\
\hline Arcilla (\%) & 14,2 & 16,2 \\
\hline \multicolumn{3}{|c|}{ Propiedades químicas de suelo: } \\
\hline $\mathrm{N}-\mathrm{NO}_{3}\left(\mathrm{mg} \mathrm{kg}^{-1}\right)$ & 30,0 & 12,5 \\
\hline $\mathrm{Na}\left(\mathrm{cmol} \mathrm{kg}^{-1}\right)$ & 1,0 & 0,5 \\
\hline Materia orgánica (\%) & 1,2 & 2,4 \\
\hline $\mathrm{pH}$ & 5,6 & 6,2 \\
\hline
\end{tabular}

Variables y análisis de dato. El diámetro basal, altura y diámetro de copa de las plantas se midieron al inicio de la plantación (a los 6 meses de edad) y dos años después del establecimiento de la plantación (en junio y julio de 2011); con estos datos se determinó el incremento periódico anual del diámetro basal, altura y diámetro de copa con la ecuación siguiente:

$$
I P A_{X}=\frac{X_{A 2}-X_{A 0}}{n}
$$

Donde: $I P A_{X}$ es el incremento periódico de la variable $X$ (diámetro basal, altura o diámetro de copa), $X_{A 2}$ es el valor de la variable $\mathrm{X}$ a dos años de la plantación, $X_{A 0}$ es el valor de la variable al momento de la plantación y $n$ es el periodo de tiempo (2 años).

El número de bifurcaciones y la supervivencia se evaluaron dos años después de la plantación, esta última como planta viva (1) o planta muerta (0).

Los supuestos de normalidad y homogeneidad de varianzas se verificaron con la prueba de Shapiro-Wilks y de Levene, respectivamente. Ninguna variable cumplió con ambos supuestos, por tanto, las diferencias entre procedencias se determinaron con pruebas no paramétricas de varianza y comparaciones múltiples de rangos (Conover 2012). El efecto de las procedencias en cada localidad se analizó con modelo siguiente:

$$
Y_{i j k}=\mu+B_{i}+P_{j}+B P_{i j+} \varepsilon_{i j k}
$$

Donde: $Y_{i j k}$ es el valor de la k-ésima observación de la jésima procedencia en el i-ésimo bloque, $\mu$ es el efecto de la media general, $B_{i}$ es el efecto del i-ésimo bloque, $P_{j}$ es el efecto de la j-ésima procedencia, $B P_{i j}$ es la interacción de bloques por procedencia y $\varepsilon_{i j k}$ es el efecto del error aleatorio.

El efecto de la localidad, la procedencia y la interacción de estos se determinó con el modelo siguiente:

$$
Y_{i j k l}=\mu+L_{i}+B_{j(i)}+P_{k}+L P_{i k}+B P_{i j k}+\varepsilon_{i j k l}
$$

Donde: $Y_{i j k l}$ es el valor del árbol (observación) establecido en la i-ésima localidad, en el j-ésimo bloque de la k-ésima procedencia; $\mu$ es el efecto de la media general; $L_{i}$ es el efecto de la i-ésima localidad; $B_{j(i)}$ es el efecto del j-ésimo bloque anidado en la i-ésima localidad; $P_{k}$ es el efecto de la k-ésima procedencia; $L P_{i k}$ es el efecto de la interacción entre la i-ésima localidad con la k-ésima procedencia; $B P_{i j k}$ es el efecto de la interacción entre el j-ésimo bloque con la k-ésima procedencia; $\varepsilon_{i j k l}$ es el efecto del error experimental.

Los componentes de varianza se estimaron con el procedimiento VARCOMP del programa estadístico SAS mediante el método de máxima verosimilitud restringida (REML). Los componentes de varianza se usaron para conocer la existencia de la interacción genotipo $\mathrm{x}$ ambiente mediante la correlación genética tipo B, la cual se determinó con la ecuación siguiente (Sierra-Lucero et al. 2002, White et al. 2007):

$$
r_{B}=\frac{\sigma_{P}^{2}}{\sigma_{P}^{2}+\sigma_{L P}^{2}}
$$

Donde: $r_{B}$ es la correlación genética tipo $\mathrm{B}, \sigma_{P}^{2}$ es el componente de varianza de la procedencia, $\sigma_{L P}^{2}$ es el componente de varianza de la interacción localidad x procedencia.

La correlación genética tipo B se calculó considerando todas las procedencias y eliminando de una hasta cuatro de ellas para observar cambios en la interacción genotipo $\mathrm{x}$ ambiente y conocer el nivel de inestabilidad de las procedencias en distintos ambientes (Salaya-Domínguez et al. 2012).

\section{RESULTADOS}

Diferencias entre localidades. El incremento periódico anual de diámetro basal, de diámetro de copa y el número de bifurcaciones fueron diferentes entre las localidades 
$(P<0,001)$, situación que no ocurrió para el incremento periódico anual de altura $(P=0,2597)$ y la supervivencia $(P=0,096)$. En la localidad Pinotepa de Don Luis el incremento periódico anual de diámetro basal fue $1,56 \mathrm{~cm}$, el de altura de $0,59 \mathrm{~m}$, el de diámetro de copa de $0,32 \mathrm{~m}$, número de bifurcaciones de 4,73 y supervivencia de $62,8 \%$. En cambio, en la localidad Valdeflores el incremento periódico anual de diámetro basal fue $0,84 \mathrm{~cm}$, el de altura de $0,54 \mathrm{~m}$, el de diámetro de copa de $0.19 \mathrm{~m}$, bifurcaciones de 2,77 y supervivencia de $54,2 \%$.

Diferencias entre procedencias. En la localidad Pinotepa de Don Luis se encontraron diferencias significativas entre procedencias para todas las variables $(P \leq 0,044)$. La procedencia local (Pinotepa de Don Luis) tuvo los valores mayores en incremento periódico anual de diámetro basal, de altura y de diámetro de copa; mientras que las procedencias Tataltepec y San Pedro tuvieron los valores mayores de bifurcaciones y supervivencia, respectivamente. En cambio, la procedencia La Tuza presentó los valores más bajos de incremento periódico anual de diámetro basal y de altura; por su parte, la procedencia Colotepec tuvo los valores menores en incremento periódico anual de diámetro de copa y bifurcaciones, y en Pinotepa de Don Luis ocurrió la supervivencia menor (cuadro 3).

En la localidad Valdeflores, para todas las variables existieron diferencias significativas entre procedencias $(P \leq 0,043)$, excepto para incremento periódico anual de diámetro de copa $(P=0,055)$. Colotepec y Pinotepa de Don Luis presentaron los valores más altos de incremento periódico anual de diámetro basal y para las demás variables fueron estadísticamente iguales con las procedencias de mayor valor. Pinotepa de Don Luis tuvo mayor número de bifurcaciones. La procedencia El Zarzal presentó incremento periódico anual de altura mayor; asimismo, Cortijo y Tataltepec presentaron supervivencia mayor (cuadro 3). Las procedencias San Pedro y Tataltepec presentaron los valores más bajos de incremento periódico anual de diámetro basal y de altura. San Francisco tuvo la menor supervivencia. En cambio, San Francisco y Los Limones presentaron menor número de bifurcaciones (cuadro 3).

Interacción genotipo $x$ ambiente. La interacción genotipo $\mathrm{x}$ ambiente fue significativa $(P \leq 0,030)$ en todas las variables. La correlación genética tipo B fue 0,000 en la mayoría de las variables cuando se consideraron todas las procedencias (10) en el análisis, mientras que el incremento periódico anual de diámetro basal tuvo un valor de 0,458 (cuadro 4). Las procedencias presentaron variación en su comportamiento en respuesta a la localidad de plantación. Para todas las variables la mayoría de las procedencias presentaron mayores valores en Pinotepa de Don Luis con relación a Valdeflores, excepto el incremento periódico anual de altura, donde solo cuatro procedencias presentaron esta tendencia (cuadro 3, figura 2). Asimismo, para todas las variables varias procedencias cambian de posición al estar plantadas en una u otra localidad (figura 2). Al eliminar en el análisis las procedencias que cambian de posición, el valor de la correlación genética tipo B se modificó para todas las variables, excepto el número de bifurcaciones (cuadro 4).

Cuadro 3. Valores promedios y comparaciones múltiples de rangos $(\alpha \leq 0,05)$ de las variables de crecimiento de árboles de Enterolobium cyclocarpum de procedencias evaluadas en Pinotepa de Don Luis (PDL) y Valdeflores (VAL), costa de Oaxaca, México.

Means and multiple rank comparisons $(\alpha \leq 0.05)$ of the growth traits of Enterolobium cyclocarpum trees from provenances evaluated in Pinotepa de Don Luis (PDL) and Valdeflores (VAL), coast of Oaxaca, Mexico.

\begin{tabular}{lcccccccccc}
\hline \multirow{2}{*}{ Procedencia } & \multicolumn{2}{c}{ IPDB $(\mathrm{cm})$} & \multicolumn{2}{c}{ IPA $(\mathrm{m})$} & \multicolumn{2}{c}{ IPDC $(\mathrm{cm})$} & \multicolumn{2}{c}{ NBIF } & \multicolumn{2}{c}{ SUP $(\%)$} \\
\cline { 2 - 10 } & PDL & VAL & PDL & VAL & PDL & VAL & PDL & VAL & PDL & VAL \\
\hline Cortijo & $1,47 \mathrm{bc}$ & $0,89 \mathrm{ab}$ & $0,46 \mathrm{~cd}$ & $0,57 \mathrm{ab}$ & $0,32 \mathrm{~b}$ & $0,20 \mathrm{a}$ & $5,00 \mathrm{a}$ & $2,82 \mathrm{ab}$ & $43,3 \mathrm{c}$ & $70,8 \mathrm{a}$ \\
Pinotepa de Don Luis & $1,94 \mathrm{a}$ & $1,06 \mathrm{a}$ & $0,82 \mathrm{a}$ & $0,47 \mathrm{ab}$ & $0,48 \mathrm{a}$ & $0,12 \mathrm{a}$ & $4,65 \mathrm{a}$ & $3,92 \mathrm{a}$ & $70,8 \mathrm{abc}$ & $50,0 \mathrm{abc}$ \\
El Zarzal & $1,63 \mathrm{ab}$ & $0,78 \mathrm{abc}$ & $0,62 \mathrm{abc}$ & $0,67 \mathrm{a}$ & $0,27 \mathrm{~b}$ & $0,14 \mathrm{a}$ & $5,40 \mathrm{a}$ & $2,40 \mathrm{~b}$ & $62,5 \mathrm{abc}$ & $62,5 \mathrm{ab}$ \\
La Tuza & $1,25 \mathrm{c}$ & $0,81 \mathrm{abc}$ & $0,44 \mathrm{~d}$ & $0,52 \mathrm{ab}$ & $0,22 \mathrm{~b}$ & $0,24 \mathrm{a}$ & $4,46 \mathrm{a}$ & $2,23 \mathrm{~b}$ & $54,2 \mathrm{bc}$ & $54,2 \mathrm{abc}$ \\
Tataltepec & $1,43 \mathrm{bc}$ & $0,72 \mathrm{bc}$ & $0,70 \mathrm{ab}$ & $0,39 \mathrm{~b}$ & $0,29 \mathrm{~b}$ & $0,20 \mathrm{a}$ & $5,50 \mathrm{a}$ & $2,76 \mathrm{~b}$ & $58,3 \mathrm{abc}$ & $70,8 \mathrm{a}$ \\
San Francisco & $1,74 \mathrm{ab}$ & $0,93 \mathrm{ab}$ & $0,53 \mathrm{~cd}$ & $0,61 \mathrm{ab}$ & $0,33 \mathrm{~b}$ & $0,24 \mathrm{a}$ & $4,64 \mathrm{a}$ & $2,00 \mathrm{~b}$ & $58,3 \mathrm{abc}$ & $33,3 \mathrm{c}$ \\
Los Limones & $1,59 \mathrm{ab}$ & $0,78 \mathrm{abc}$ & $0,59 \mathrm{bc}$ & $0,53 \mathrm{ab}$ & $0,31 \mathrm{~b}$ & $0,20 \mathrm{a}$ & $5,11 \mathrm{a}$ & $2,00 \mathrm{~b}$ & $72,2 \mathrm{abc}$ & $45,8 \mathrm{abc}$ \\
San Pedro & $1,51 \mathrm{bc}$ & $0,62 \mathrm{c}$ & $0,56 \mathrm{bcd}$ & $0,43 \mathrm{~b}$ & $0,34 \mathrm{~b}$ & $0,12 \mathrm{a}$ & $4,53 \mathrm{a}$ & $2,53 \mathrm{~b}$ & $83,3 \mathrm{a}$ & $62,5 \mathrm{ab}$ \\
Colotepec & $1,34 \mathrm{bc}$ & $1,04 \mathrm{a}$ & $0,47 \mathrm{~cd}$ & $0,64 \mathrm{a}$ & $0,19 \mathrm{~b}$ & $0,24 \mathrm{a}$ & $2,91 \mathrm{~b}$ & $3,38 \mathrm{a}$ & $45,8 \mathrm{bc}$ & $54,2 \mathrm{abc}$ \\
Pochutla & $1,70 \mathrm{ab}$ & $0,76 \mathrm{abc}$ & $0,70 \mathrm{ab}$ & $0,54 \mathrm{ab}$ & $0,41 \mathrm{a}$ & $0,20 \mathrm{a}$ & $5,11 \mathrm{a}$ & $3,67 \mathrm{a}$ & $79,2 \mathrm{ab}$ & $37,5 \mathrm{bc}$ \\
\hline
\end{tabular}

IPDB: incremento periódico anual de diámetro basal, IPA: incremento periódico anual de altura, IPDC: incremento periódico anual de diámetro de copa, NBIF: número de bifurcaciones, SUP: supervivencia. Letras diferentes en cada columna indican diferencias con $P \leq 0,05$. 

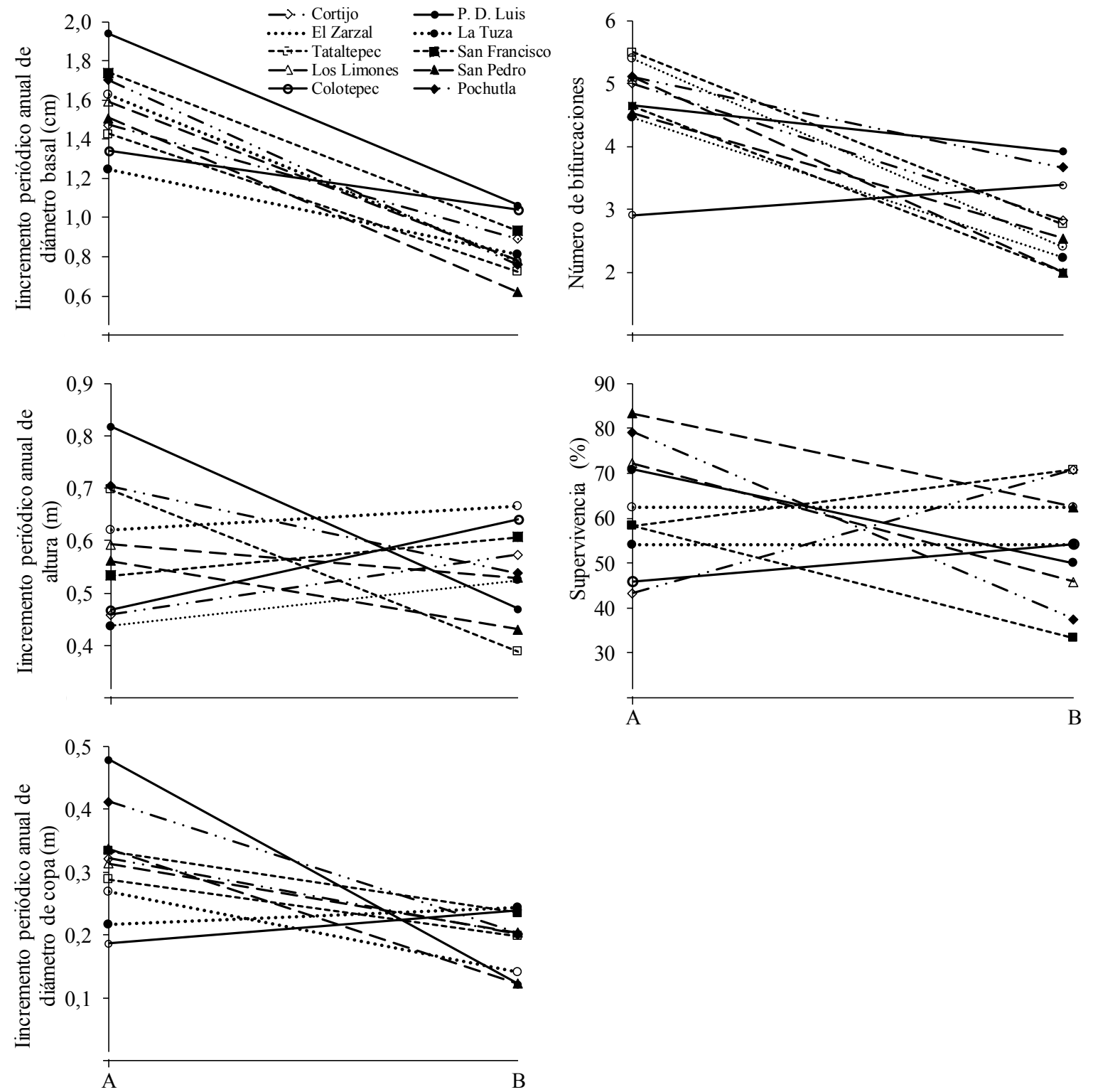

Figura 2. Incremento periódico anual de altura, diámetro basal, diámetro de copa, número de bifurcaciones y supervivencia de Enterolobium cyclocarpum en dos localidades (A: Pinotepa de Don Luis, B: Valdeflores) en la costa de Oaxaca, México.

Annual periodic increment in height, basal diameter, crown diameter, number of bifurcations and survival of Enterolobium cyclocarpum in two sites (A: Pinotepa de Don Luis, B: Valdeflores) on the coast of Oaxaca, Mexico. 
Cuadro 4. Valores de correlación genética tipo B de las variables de crecimiento incluyendo todas y excluyendo algunas procedencias de Enterolobium cyclocarpum ensayadas en la costa de Oaxaca, México.

Type B genetic correlation values of the growth traits where all provenances were included and some provenances were excluded of Enterolobium cyclocarpum tested on the coast of Oaxaca, Mexico.

\begin{tabular}{cccccc}
\hline NPE & IPDB & IPA & IPDC & NBIF & SUP \\
\hline 0 & 0,458 & 0,000 & 0,000 & 0,000 & 0,000 \\
1 & 1,000 & 0,000 & 0,000 & 0,000 & 0,000 \\
2 & 1,000 & 0,163 & 0,000 & 0,000 & 1,000 \\
3 & 1,000 & 0,194 & 0,000 & 0,000 & 1,000 \\
4 & 1,000 & 0,318 & 1,000 & 0,000 & 1,000 \\
\hline
\end{tabular}

NPE: número de procedencias excluidas en el análisis $(0=$ ninguna procedencia excluida, $1=$ una procedencia excluida, ..., $4=$ cuatro procedencias excluidas), IPDB: incremento periódico anual de diámetro basal, IPA: incremento periódico anual de altura, IPDC: incremento periódico anual de diámetro de copa, NBIF: número de bifurcaciones, SUP: supervivencia.

\section{DISCUSIÓN}

Diferencias entre localidades. Las diferencias entre las localidades Pinotepa de Don Luis y Valdeflores en número de bifurcaciones y el incremento periódico anual de diámetro basal y del diámetro de copa de plantas de E. cyclocarpum se atribuyen a diferencias ambientales relacionadas con su ubicación geográfica y el tipo de suelo. Entre las localidades, la diferencia es $365 \mathrm{~m}$ de elevación, $629 \mathrm{~mm}$ de precipitación media anual y $0,7{ }^{\circ} \mathrm{C}$ de temperatura media anual. Pinotepa de Don Luis presenta mejores condiciones ambientales y de suelo que Valdeflores. El efecto asociado de la altitud que se encontró para E. cyclocarpum es similar al efecto que se reporta en otras especies. Por ejemplo, plantas de Pinus pseudostrobus Lindl. presentan crecimientos iniciales mayores en localidades ubicadas a mayor altitud (Viveros-Viveros et al. 2005).

La diferencia en precipitación de las localidades, asociado a la diferencia altitudinal, ocasiona diferencias de humedad. En Pinotepa de Don Luis el índice de aridez es menor que en Valdeflores (cuadro 2), lo que hace que Valdeflores sea más seco. Esta diferencia de humedad parece determinar las diferencias que existen en este estudio; por ejemplo, Schenone et al. (2002) reportan que el déficit hídrico tiene efectos negativos en la supervivencia, diámetro y altura de Eucalyptus dunnii Maiden. Asimismo, las diferencias en altura y diámetro basal de Acacia saligna (Labill.) H. L. Wendl en dos sitios de plantación se atribuyen a las diferencias hídricas entre los sitios (Mora-Poblete y Meneses-Rojas 2004).

Las diferencias en crecimiento de Hymenaea courbaril L., Swietenia macrophylla King, Cedrela odorata L., Erisma uncinatum Warm, Cordia alliodora (Ruíz et Pav.)
Oken. y Tabebuia serratifolia (Vahl) Nicholson se atribuyen a discrepancias en las características del suelo de los sitios de plantación (Lozada et al. 2003). Por lo anterior, las diferencias que se encontraron entre localidades para E. cyclocarpum puede deberse también a las propiedades físicas y condiciones de fertilidad del suelo. El suelo en Pinotepa de Don Luis tiene cantidades mayores de $\mathrm{N}_{-} \mathrm{NO}_{3}$ y Na que el suelo de Valdeflores (cuadro 2); por el contrario, el suelo de Valdeflores tiene mayor materia orgánica y pH que el suelo de Pinotepa de Don Luis (cuadro 2). Craven et al. (2007) reportan diferencias en productividad de E. cyclocarpum y de otras especies tropicales debidas a diferencias en fertilidad y precipitación de los sitios de plantación. Esto también coincide parcialmente con lo que reporta Valencia-Manzo et al. (2006) para Pinus greggii Engelm., donde el $\mathrm{pH}$ menor, contenido de materia orgánica mayor y porcentaje de nitrógeno mayor favorecen el crecimiento en diámetro basal, altura total, diámetro de copa y número de ciclos de crecimiento. Para E. cyclocarpum, la fertilidad de suelos y las condiciones climáticas de los sitios, además de influir en el crecimiento de las plantas, también definen en el contenido nutrimental de las semillas (Barrientos-Ramírez et al. 2015).

Vázquez-Yanes et al. (1999) mencionan que E. cyclocarpum presenta mejor desarrollo en suelos vertisoles de textura arenoso-arcilloso y arenoso, contrario al resultado del presente estudio. En Pinotepa de Don Luis, donde existen mayores crecimientos de E. cyclocarpum, los suelos contienen menores porcentajes de arena y arcilla, pero mayor porcentaje de limo que los suelos de Valdeflores (cuadro 2). Estas características físicas pueden ocasionar que los suelos en Valdeflores conserven menos humedad en la época de sequía; por ejemplo, en enero del 2011, el contenido de humedad de suelo en Valdeflores fue 3,3\%, mientras que en Pinotepa de Don Luis fue 8,0\%.

El incremento periódico anual de altura y la supervivencia son similares entre localidades, posiblemente debido a otros factores como plagas y fauna, los cuales influyen en estas variables. Por ejemplo, en ambas localidades hongos del género Oidium afectaron las yemas principales, lo que influye en el crecimiento en altura. Aunque, E. cyclocarpum es tolerante a la sequía (Foroughbakhch et al. 2006), esta afectó mayormente la supervivencia de las plantas en Valdeflores, mientras que las tuzas (Geomydae) disminuyeron mayormente la supervivencia en Pinotepa de Don Luis. Las tuzas se alimentan de la raíz principal de las plantas de E. cyclocarpum, ocasionándoles la muerte. Leopold et al. (2001) reportan bajos crecimientos de E. cyclocarpum debido al ataque de herbívoros en Costa Rica; mientras que Viveros-Viveros et al. (2005) reportan daños por roedores en $P$. pseudostrobus debido al uso agrícola que tuvo el sitio de plantación, al igual que Pinotepa de Don Luis.

Diferencias entre procedencias. En la localidad Pinotepa de Don Luis la procedencia local de E. cyclocarpum muestra ventajas mayores por estar más adaptada al sitio de 
plantación, mientras que el uso de procedencias provenientes de altitudes inferiores (La Tuza y Colotepec) provoca crecimientos menores. El efecto de la altitud también fue evidente en la supervivencia de E. cyclocarpum, las plantas de Cortijo (procedencia localizada a $37 \mathrm{~m}$ de elevación y a $400 \mathrm{~m}$ de desnivel del sitio de plantación) tienen la supervivencia más baja. Para que el crecimiento y la supervivencia de E. cyclocarpum no sean afectadas por inadaptación, las plantas se deben establecer hasta $\pm 105 \mathrm{~m}$ altitudinales a partir de la altitud del sitio de recoleta de semillas (QuinoPascual 2013). Otros estudios demuestran desempeños similares de estas procedencias; por ejemplo, la procedencia Pinotepa de Don Luis presenta valores mayores en parámetros germinativos, mientras que Cortijo y La Tuza presentan valores más bajos (Viveros-Viveros et al. 2017).

En la localidad Valdeflores (de baja elevación), la procedencia Colotepec (la más cercana al sitio de plantación) junto con El Zarzal, La Tuza y Cortijo, que proceden de altitudes bajas, presentan los crecimientos y supervivencia mayores. Lo anterior es contrario a lo encontrado en la localidad Pinotepa de Don Luis, que es de mayor elevación. Por otra parte, las procedencias con crecimientos y supervivencia mayores tienen índices de aridez similares $(0,09$ a 0,11$)$ al de la localidad de plantación $(0,11)$ (ViverosViveros et al. 2017). La procedencia Pinotepa de Don Luis plantada en Valdeflores presenta respuesta adecuada en cuanto al incremento de diámetro basal, pero tiene mayor número de bifurcaciones, por lo que resulta inadecuado plantar dicha procedencia en sitios con condiciones similares a Valdeflores, ya que las bifurcaciones generan madera de menor calidad.

Las diferencias en crecimiento en cada localidad también pueden deberse a adaptaciones genéticas de las procedencias a ambientes diferentes (López-Upton et al. 2004) de la región Costa de Oaxaca, México. En cada localidad las procedencias parecen seguir estrategias adaptativas diferentes; así, en Pinotepa de Don Luis las procedencias con mayor productividad probablemente realizan mayor actividad fotosintética, como resultado de mayor precipitación y fertilidad del suelo, mientras que en Valdeflores las procedencias con mayor éxito parecen incrementar su eficiencia en el uso del agua y decrecen su capacidad fotosintética neta como resultado de niveles de fertilidad y precipitación bajos (Craven et al. 2007).

Cuando se trata de identificar las mejores procedencias para cierta región, es imprescindible comparar los resultados del ensayo con los obtenidos en otras regiones. Lo anterior porque en caso de obtener resultados deficientes con las procedencias evaluadas, es posible detectar procedencias de otras regiones para probarlas en la región de interés. En este sentido, en la localidad Pinotepa de Don Luis los incrementos periódicos anuales de diámetro basal y de altura de la mayoría de las procedencias fueron mayores o similares a lo reportado en plantaciones de Nayarit $(1,27$ $\mathrm{cm})$, Nuevo León $(2,25 \mathrm{~cm})$ y Colima $(1,03 \mathrm{a} 1,11 \mathrm{~cm})$, México (Makocki y Valdez-Hernández 2001, Fo- roughbakhch et al. 2006, Jiménez-Pascual 2011), México; por el contrario, en Valdeflores, sin demeritar el desempeño de las procedencias más cercanas, la mayoría tuvieron valores inferiores. En cuanto a la supervivencia, en ambas localidades, la mayoría de las procedencias tuvieron menores valores a lo reportado en Nuevo León $(73 \% \pm 7,1)$ y Colima (82 a 92 \%), México (Foroughbakhch et al. 2006, Jiménez-Pascual 2011). La supervivencia alta en Colima se debe a buenos suelos en los sitios de plantación y a la fertilización de la plantación (Jiménez-Pascual 2011). La localidad Valdeflores no es fértil por sus suelos someros y arenosos. En cambio, a pesar de los suelos profundos y fértiles en Pinotepa de Don Luis, la supervivencia fue mermada por los daños causados por roedores de la familia Geomydae.

Las mejores condiciones ambientales y de suelos en Pinotepa de Don Luis posiblemente favorecen al incremento periódico anual de diámetro basal de todas las procedencias, ya que todas tienen valores superiores a los crecimientos anuales de diámetro normal (medido a $1,30 \mathrm{~m}$ ) de todas las categorías diamétricas de E. cyclocarpum en selvas secas del estado de Gurrero, México (Pineda-Herrera et al. 2017). Es importante recalcar que los incrementos de diámetro basal obtenidos a edad temprana son altos y pueden ser mucho mayores a edades superiores, esto debido a que $E$. cyclocarpum tiene crecimiento mayor conforme incrementa su edad (Pineda-Herrera et al. 2017). Por otra parte, a pesar de que las condiciones ambientales y de suelo son restrictivas en la localidad Valdeflores, el incremento periódico anual de diámetro basal de todas procedencias es superior al crecimiento de $E$. cyclocarpum con categoría diamétrica de 5,10 y $15 \mathrm{~cm}$ de poblaciones naturales del estado de Guerrero, México (Pineda-Herrera et al. 2017).

El crecimiento de diámetro de copa y las bifurcaciones son parámetros poco evaluados en etapa inicial de plantaciones de E. cyclocarpum. El crecimiento de diámetro de copa es importante en la selección de procedencias para plantaciones con fines forrajeros y de protección al suelo, ya que esta variable se correlaciona con la producción de follaje. Es importante evaluar las bifurcaciones porque esta característica es indeseable, debido a que los árboles bifurcados tienen nula o escasa posibilidad de aserrío y el problema es más severo cuando la bifurcación ocurre a menor altura del fuste y es más abundante. Por tanto, si el objetivo es la producción de madera para la industria de aserrío, las procedencias de E. cyclocarpum con más bifurcaciones serán menos productivas y con madera de calidad baja. Al respecto, la procedencia Colotepec de origen más oriental respecto a la localidad de plantación (Pinotepa de Don Luis) tiene mejor respuesta (menos bifurcaciones); por el contrario, Cortijo y Pinotepa de Don Luis de origen más occidental respecto a la localidad de plantación (Valdeflores) tienen mejor respuesta. Lo anterior podría ser resultado de un posible efecto del movimiento longitudinal, asociado con la precipitación y otros factores no considerados en esta investigación. Por ejemplo, la proporción 
alta de árboles bifurcados de Tectona grandis L.f. puede deberse la inundación (Chaix et al. 2011); en cambio, en Khaya senegalensis (Desr.) A. Juss. la ramificación múltiple está correlacionada con los daños por plagas (Peng et al. 2010). En E. cyclocarpum, hongos del género Oidium ocasionan la muerte de las yemas principales y estimulan la formación de brotes axilares de E. cyclocarpum quienes posiblemente favorecen la formación de bifurcaciones.

Interacción genotipo $x$ ambiente. La interacción genotipo $\mathrm{x}$ ambiente se reporta tanto en especies latifoliadas como en coníferas (López-Upton et al. 2004, Mora-Poblete y Meneses-Rojas 2004). Sin embargo, contrario a E. cyclocarpum, algunas especies no presentan interacción genotipo $\mathrm{x}$ ambiente en supervivencia, diámetro, altura y ciclos de crecimiento, (Viveros-Viveros et al. 2005, ValenciaManzo et al. 2006).

Cuando existe interacción genotipo $\mathrm{x}$ ambiente, la variabilidad es considerable en los ensayos genéticos debido a que los fenotipos responden de manera diferente en cada ambiente (Mora-Poblete y Meneses-Rojas 2004). Por lo anterior, la existencia de interacción genotipo x ambiente en E. cyclocarpum a edad juvenil dificulta la selección temprana de una procedencia para varios sitios de plantación; sin embargo, se deben realizar evaluaciones posteriores porque a mayor edad de los árboles, la interacción genotipo $\mathrm{x}$ ambiente puede no ser significativa para algunas variables (Salaya-Domínguez et al. 2012). Asimismo, de persistir la interacción genotipo $\mathrm{x}$ ambiente a edad mayor será necesario seleccionar una $\mathrm{o}$ un grupo de procedencias para cada sitio específico para obtener rendimientos mayores (Mora-Poblete y Meneses-Rojas 2004). En Pinus taeda L. la correlación genética tipo B aumenta a edades mayores, lo que indica que el efecto de la interacción genotipo x ambiente disminuye (Sierra-Lucero et al. 2002). Los valores bajos de correlación genética tipo B para $E$. cyclocarpum indican interacción genotipo $\mathrm{x}$ ambiente alta para todas las variables (Sierra-Lucero et al. 2002, White et al. 2007), excepto para el crecimiento en diámetro basal que presentó valor moderado. La especificidad de ciertas procedencias al ambiente es diferente para cada variable; por ejemplo, la interacción es moderadamente importante (White et al. 2007), ya que una, dos y cuatro procedencias son interactivas para el crecimiento en diámetro basal, supervivencia y crecimiento en diámetro de copa, respectivamente. Por otro lado, para el crecimiento en altura y número de bifurcaciones, aún con la exclusión de hasta cuatro procedencias la interacción es de suma importancia biológica (White et al. 2007), lo que indica inestabilidad mayor de las procedencias para estas variables, lo cual es común en árboles o procedencias silvestres (Salaya-Domínguez et al. 2012). Debido a la inestabilidad de las procedencias probadas, la selección de estas para establecer plantaciones dependerá del ambiente de establecimiento (Salaya-Domínguez et al. 2012) y del objetivo de la plantación. El uso de procedencias locales o cercanas a los sitios de plantación es recomendable, y es necesario considerar desplazamientos no mayores a $105 \mathrm{~m}$ en altitud (Quino-Pascual 2013).

\section{CONCLUSIONES}

El crecimiento de las procedencias de Enterolobium cyclocarpum es diferente en cada localidad evaluada, como consecuencia de las diferencias ambientales y de suelo en cada una de estas, por lo que es importante considerar el origen del germoplasma y las condiciones ambientales de las localidades donde se establecerán plantaciones con esta especie.

En general, para las variables de crecimiento, en la localidad Pinotepa de Don Luis se observan resultados más favorables que en Valdeflores, lo cual no sucede para la supervivencia, porque esta presenta influencia de un factor biótico.

Las plantas originadas con semilla de procedencias cercanas a las localidades de evaluación presentan crecimientos mayores, por lo que se recomienda el uso de procedencias locales o cercanas a los sitios de plantación.

Las plantas de procedencias que presentan tasas mayores de crecimientos o tasas menores de crecimiento en un sitio de evaluación son diferentes en el otro sitio de evaluación, por lo que se confirma la influencia de la interacción genotipo $\mathrm{x}$ ambiente en el crecimiento y supervivencia de las plantas de E. cyclocarpum.

\section{AGRADECIMIENTOS}

Los autores agradecen a Apolonio Galán Hernández y Justino Ríos por proporcionar los terrenos para establecer las plantaciones, así como a René Robles Silva por la recolecta de semillas.

\section{REFERENCIAS}

Aldama-Barajas R, M Aguilera-Rodríguez. 2003. Procedimientos y cálculos básicos, útiles en la operación de viveros que producen en contenedor. Guadalajara, México. CONAFOR. 45 p. URL: https://bibliotecadigital. infor.cl/bitstream/handle/20.500.12220/17398/25107. pdf? sequence $=1 \&$ isAllowed $=\mathrm{y}$

Barrientos-Ramírez L, JJ Vargas-Radillo, M Segura-Nieto, R Manríquez-González, FA López-Dellamary. 2015. Nutritional evaluation of mature seeds of Enterolobium cyclocarpum (parota) from diverse ecological zones in western Mexico. Bosque 36(1): 95-103. DOI: 10.4067/S0717$\underline{92002015000100010}$

Chaix G, O Monteuuis, C Garcia, D Alloysius, J Gidiman, R Bacilieri, DKS Goh. 2011. Genetic variation in major phenotypic traits among diverse genetic origins of teak (Tectona grandis L.f.) planted in Taliwas, Sabah, East Malaysia. Annals of Forest Science 68(5): 1015-1026. DOI: $10.1007 /$ s13595-011-0109-8

Conover WJ. 2012. The rank transformation-an easy and intuitive way to connect many nonparametric methods to their 
parametric counterparts for seamless teaching introductory statistics courses. Computational Statistics 4(5): 432-438. DOI: $10.1002 /$ wics. 1216

Craven D, D Braden, MS Ashton, GP Berlyn, M Wishnie, D Dent. 2007. Between and within-site comparisons of structural and physiological characteristics and foliar nutrient content of 14 tree species at a wet, fertile site and a dry, infertile site in Panama. Forest Ecology and Management 238: 335-346. DOI: 10.1016/j.foreco.2006.10.030

Foroughbakhch R, MA Alvarado-Vázquez, JL Hernández-Piñero, A Rocha-Estrada, MA Guzmán-Lucio, EJ Treviño-Garza. 2006. Establishment, growth and biomass production of 10 tree woody species introduced for reforestation and ecological restoration in northeastern Mexico. Forest Ecology and Management 235: 194-201. DOI: 10.1016/j.foreco.2006.08.012

Jiménez-Pascual JL. 2011. Evaluación de plantaciones forestales comerciales de parota Enterolobium cyclocarpum (Jacq.) Griseb., en el estado de Colima. Tesis de Licenciatura. Uruapan, México. Facultad de Agrobiología, Universidad Michoacana de San Nicolás de Hidalgo. 55 p. URL: http://bibliotecavirtual.dgb.umich.mx:8083/jspui/handle/123456789/131

Leopold AC, R Andrus, A Finkeldey, D Knowles. 2001. Attempting restoration of wet tropical forests in Costa Rica. Forest Ecology and Management 142: 243-249. DOI: 10.1016/ S0378-1127(00)00354-6

López-Upton J, C Ramírez-Herrera, O Plascencia-Escalante, J Jasso-Mata. 2004. Variación en crecimiento de diferentes poblaciones de las dos variedades de Pinus greggii. Agrociencia 38(4): 457-464. URL: https://www.colpos.mx/ agrocien/Bimestral/2004/jul-ago/art-9.pdf

Lozada JR, J Moreno, R Suescun. 2003. Plantaciones en fajas de enriquecimiento. Experiencias en 4 Unidades de Manejo Forestal de la Guayana Venezolana. Interciencia 28(10): 568-575. URL: https://www.redalyc.org/articulo. oa? id $=33908502$

Makocki MW, JI Valdez-Hernández. 2001. Radial growth periodicity of tree species in a semi-deciduous tropical forest of the Pacific coast of México: Implications for their management. In Proceedings of the IUFRO joint symposium on tree seed technology, physiology and tropical silviculture. Los Baños, Philippines. University of the Philippines. p. 85-96. URL: https://www.researchgate.net/publication/264975223 Radial growth periodicity of tree species in a semi-deciduous tropical forest of the Pacific coast of Mexico Implications for their management

Mora-Poblete F, R Meneses-Rojas. 2004. Comportamiento de procedencias de Acacia saligna (Labill.) H. L. Wendl. en la región de Coquimbo, Chile. Ciência Forestal, Santa María 14(1): 103-109. DOI: 10.5902/198050981786

Olivares-Pérez J, F Avilés-Nova, B. Albarrán-Portillo, S RojasHernández, OA Castelán-Ortega. 2011. Identificación, usos y medición de leguminosas forrajeras en ranchos ganaderos del sur del Estado de México. Tropical and Subtropical Agroecosystems 14(2): 739-748. URL: http://www.redalyc. org/pdf/939/93918231039.pdf

Peng R, K Christin, D Rilly. 2010. Weaver ants, Oecophylla smaragdina (Hymenoptera: Formicidae), as biocontrol agents on African mahogany trees, Khaya senegalensis (Sapindales: Meliaceae), in the Northern Territory of Australia. In- ternational Journal of Pest Management 56(4): 363-370. DOI: $10.1080 / 09670874.2010 .503286$

Pennington TD, J Sarukhán. 2005. Árboles tropicales de México. Manual para la identificación de las principales especies. Ciudad de México, México. UNAM. 523 p.

Pineda-Herrera E, JI Valdez-Hernández, CP Pérez-Olvera, R Dávalos-Sotelo. 2017. Fenología, incremento en diámetro y periodicidad de anillos de crecimiento de Enterolobium cyclocarpum (Jacq.) Griseb (Leguminoceae) en Costa Grande, Guerrero, México. Polibotanica 43: 1-21. DOI: $10.18387 /$ polibotanica.43.9

Quino-Pascual K. 2013. Germinación de semillas y crecimiento inicial de plántulas de Enterolobium cyclocarpum (Jacq.) Griseb. procedentes de la costa de Oaxaca. Tesis de Licenciatura. Xalapa, México. Facultad de Ciencias Agrícolas, Universidad Veracruzana. 68 p.

Salas-Morales SH, A Saynes-Vázquez, L Schibli. 2003. Flora de la Costa de Oaxaca, México: lista florística de la región de Zimatán. Boletín de la Sociedad Botánica de México 72: 21-58. DOI: $10.17129 /$ botsci.1669

Salaya-Domínguez JM, J López-Upton, JJ Vargas-Hernández. 2012. Variación genética y ambiental en dos ensayos de progenies de Pinus patula. Agrociencia 46(5): 519-534. URL: https://www.colpos.mx/agrocien/Bimestral/2012/julago/art-9.pdf

Schenone RA, L Storck, MP Martins-Corder. 2002. Variación genética en procedencias/progenies de polinización abierta de Eucalyptus dunnii Maiden. Bosque 23(1): 79-89. URL: http://mingaonline.uach.cl/pdf/bosque/v23n1/art07.pdf

SEMARNAT (Secretaría de Medio Ambiente y Recursos Naturales, MX). 2002. Norma Oficial de la Mexicana NOM021-RECNAT-2000 que establece las especificaciones de fertilidad, salinidad y clasificación de suelos, estudio, muestreo y análisis. México, D. F. Diario Oficial de la Federación. 85 p. URL: http://biblioteca.semarnat.gob.mx/ janium/Documentos/Ciga/libros2009/DO2280n.pdf

Sierra-Lucero V, SE McKeand, DA Huber, DL Rockwood, TL White. 2002. Performance differences and genetic parameters for four coastal provenances of loblolly pine in the southeastern United States. Forest Science 48(4): 732-742. DOI: $10.1093 /$ forestscience/48.4.732

Valencia-Manzo S, MV Velasco-García, M Gómez-Cárdenas, M. Ruiz-Muñoz, MA Capó-Arteaga. 2006. Ensayo de procedencias de Pinus greggii Engelm. en dos localidades de la Mixteca alta de Oaxaca, México. Revista Fitotecnia Mexicana 29(1): 27-32. URL: https://www.revistafitotecniamexicana.org/documentos/29-1/4a.pdf

Vázquez-Yanes C, AI Batis-Muñoz, MI Alcocer-Silva, M Gual-Díaz, C Sánchez-Dirzo. 1999. Árboles y arbustos potencialmente valiosos para la restauración ecológica y la reforestación. Ciudad de México, México. CONABIO. 311 p. (Reporte Técnico del Proyecto J084). URL: http://www. conabio.gob.mx/institucion/proyectos/resultados/J084 Fichas $\% 20 \mathrm{de} \% 20$ Especies.pdf

Viveros-Viveros H, C Sáenz-Romero, J López-Upton, JJ VargasHernández. 2005. Variación genética altitudinal en el crecimiento de plantas de Pinus pseudostrobus Lindl. en campo. Agrociencia 39(5): 575-587. URL: https://www.colpos.mx/ agrocien/Bimestral/2005/sep-oct/art-11.pdf

Viveros-Viveros H, JD Hernández-Palmeros, MV Velasco-García, R Robles-Silva, C Ruiz-Montiel, A Aparicio-Rentería, 
MJ Martínez-Hernández, J Hernández-Villa, ML Hernández-Hernández. 2015. Análisis de semilla, tratamientos pregerminativos de Enterolobium cyclocarpum (Jacq.) Griseb. y su crecimiento inicial. Revista Mexicana de Ciencias Forestales 6(30): 52-65. DOI: 10.29298/rmcf.v6i30.207

Viveros-Viveros H, K Quino-Pascual, MV Velasco-García, G Sá-
nchez-Viveros, E Velasco-Bautista. 2017. Variación geográfica de la germinación en Enterolobium cyclocarpum en la costa de Oaxaca, México. Bosque 38(2): 317-326. DOI: $10.4067 /$ S0717-92002017000200009

White TL, TW Adams, DB Neale. 2007. Forest Genetic. Cambridge, USA. CAB International. 682 p.

Recibido: $12 / 06 / 18$

Aceptado: 06/05/19 
